\title{
Domesticated DNA transposon proteins mediate retrotransposon control
}

\author{
Kathryn A O’Donnell ${ }^{1,2}$, Jef D Boeke ${ }^{1,2}$ \\ ${ }^{I}$ Department of Molecular Biology and Genetics; ${ }^{2}$ The High Throughput Biology Center, The Johns Hopkins University School of \\ Medicine, 733 N.Broadway, Baltimore, MD 21205, USA.jboeke@jhmi.edu \\ Cell Research (2008) 18:331-333. doi: 10.1038/cr.2008.34; published online 3 March 2008
}

The Schizosaccharomyces pombe genome, like those of many eukaryotes, contains a number of retrotransposable repeat sequences. The pombe elements, termed Tf1 (transposon of fission yeast 1) and Tf2 possess long terminal repeats (LTRs) and belong to the gypsy family of retrotransposons [1]. This class of potentially deleterious mobile elements inserts at new genomic locations by reverse transcription of an RNA intermediate. These and other mobile elements have a significant impact on gene structure and influence gene expression. Therefore, S. pombe is under robust selective pressure to limit rampant transposition.

Several modes of transposon control exist in different organisms. DNA methylation is one important mechanism demonstrated to regulate transposon activity in plants, mammals, and fungi. Additionally, the RNA interference (RNAi) pathway is known to silence transposons in plants and in C. elegans. However, the RNAi machinery has a surprisingly minor role in silencing transposable elements in $S$. pombe. In fission yeast, the RNAi machinery represses transcription through recruitment of histone-modifying enzymes to chromatin. This occurs when the heterochromatin machinery targets certain classes of repeats at centromeres, telomeres, the mat locus, and rDNA [2]. Each of these regions contains dg and dh repeats which are transcribed and provide an abundant source of siRNAs that are generated by RNAi effector proteins. Hansen and colleagues tested the effects of RNAi and histone deacetylase mutations on the expression of Tf2 retrotransposons [3]. Tf2 mRNA expression was not affected by RNAi mutants. In contrast, Tf2 mRNA expression increased in the class II histone deacetylase $c l r 3 \Delta$ and the class I histone deacetylase $\operatorname{clr} 6 \Delta$ mutants, suggesting RNAi-independent regulation of Tf 2 chromatin structure. In the current study, Grewal and colleagues further investigated RNAi-independent mechanisms that regulate transposon activity [4].

Throughout evolution, a protein may be co-opted or exapted for the benefit of the host organism, whereby an existing biological feature will be adapted for a novel purpose. In nature, there are several examples in which mobile DNA element proteins provided a source for new cellular functions [5]. One wellknown example of host domestication of a transposon-encoded protein is human CENP-B, which is derived from the transposase of pogo DNA transposons. This protein binds to repeats within alpha satellite DNA and facilitates the formation of centromeres. The human protein is highly conserved and has orthologues in other systems. Three CENP-B homologues are present in $S$. pombe and are encoded by Abp1, Cbh1, and Cbh2. While they have been shown to have redundant roles in centromeric heterochromatin formation and chromosome segregation, the Abp1 null mutant exhibits a slow growth phenotype that is exacerbated by the loss of other CENP$B$ proteins, suggesting there might be additional functions for these proteins in $S$. pombe. In an ironic twist, the work of Cam et al. suggests that these transposase-derived proteins have been recruited to silence retrotransposons.

To further understand the significance of the distinct phenotypes of the CENP-B mutants, Cam et al. first mapped the chromosomal distribution of these factors using chromatin immunoprecipitation followed by microarray (ChIP-chip) analysis. Consistent with earlier studies, Abp1 was found at ARS sites and centromeres [6-8]. Surprisingly, both Abp1 and Cbh1 were also seen to be enriched at $5^{\prime}$ and $3^{\prime}$ LTRs of $\mathrm{Tf} 2$ retrotransposons. These proteins were also detected at solo LTRs, as well as other sequences not related to retrotransposons.

The authors then asked whether loss of CENP-B homologues influenced the expression of these target loci. Interestingly, Tf2 expression increased by more than 10-fold in the abpl $1 \Delta$ strain. Loss of Abp1 also increased expression of wtf elements (a type of repeat often adjacent to Tf element LTRs) and genes associated with LTRs. While the loss of Cbh1 or Cbh2 alone did not alter Tf 2 expression, deletion of one or both of these genes 
in the $a b p 1 \Delta$ cells resulted in further increases in Tf 2 expression. Grewal and colleagues therefore suggest a model in which Abp1 may recruit Cbh1 and Cbh2 to Tf2 retrotransposons. As one might predict from this model, Cbh1 binding was not detected at Tf2 elements in abp $1 \Delta$ cells, while Abp1 binding to Tf2 persisted in the $c b h 1 \Delta c b h 2 \Delta$ cells.

As discussed earlier, the histone deacylases (HDACs) Clr3, a recently identified component of the Snf2/Hdaccontaining repressor complex (SHREC), and Clr6 were previously demonstrated to control Tf2 expression. In this study, Cam et al. investigated the potential genetic interaction between these HDACs and Abp1 in Tf2 silencing. Notably, the increase in Tf2 expression in double clr $3 \Delta$ clr $6 \Delta$ mutant cells was less than that observed in abp $1 \Delta$ cells or in cells deficient in Abp1 and either Clr3 or Clr6. These results suggest that Abp1 may act upstream of the HDACS and function to recruit the Clr3 and Clr6 HDACs to silence Tf2. ChIP analysis showed that the binding of Abp 1 to Tf2 was unaffected in the double HDAC mutant. However Cbh1 binding at Tf2 was decreased in HDAC mutant cells, suggesting that $\mathrm{Clr} 3$ and Clr6 may aid in the recruitment of Cbh1 to Tf2 LTRs.

The authors then asked whether Abp1-mediated retrotransposon silencing was affected by the histone chaperone HIRA/Hip1, also implicated in Tf2 silencing [9]. Tf2 expression was significantly higher in the abp $1 \Delta$ hip $1 \Delta$ cells relative to either of the single mutants, which implies that these gene products silence Tf2 through distinct pathways.

Perhaps one of the most intriguing findings of this study is the observation that Tf2 elements cluster into higher order structures, which the authors termed 'Tf bodies'. Immunostaining analysis with Myc-tagged CENP-Bs revealed that these proteins displayed a previously unrecognized complex 'network' structure in the nucleus and near the nucleolus. In order to deter- mine whether Tf2 elements were also organized into these structures, Grewal and colleagues performed FISH analysis using probes specific for the Tf2 coding region. Although there are 13 full-length Tf2 elements in S. pombe, only 1-2 Tf2 foci were detected per nucleus in the majority of WT cells, suggesting that dispersed elements cluster together in discrete foci. Consistent with this model, three or more of the Tf2 spots were seen in CENP-B mutant strains although it is puzzling why all 13 elements were not detected. The authors conclude that CENP-Bs may indeed have a role in organizing Tf2 elements into these specialized structures. As the authors point out, Tf bodies are reminiscent of Drosophila gypsy retrotransposons, which are ordered into specialized "insulator" bodies to facilitate chromatin organization [10]. Indeed, future studies are necessary to better understand the functional role of these Tf bodies in normal cells and in response to environmental stresses. What other proteins localize to and/or regulate the organization of $\mathrm{Tf}$ bodies? Despite these open questions, these findings support a conserved role for transposable elements in regulating higher order genomic architecture.

In addition to $\mathrm{Tf} 2$ retrotransposons, the $S$. pombe genome also contains many LTR fragments and solo LTRs, a significant fraction of which belong to the extinct Tf1 element family. Because the ChIP-chip analysis revealed that Abp1 and Cbh1 were also bound to Tf1 LTRs, the authors reasoned that CENP-B proteins could also target extinct Tfl elements. They tested this hypothesis by introducing a full-length Tfl element into the genome. Previous studies had shown that Tf1 elements mobilize by integrase-mediated transposition or homologous recombination [11]. Interestingly, a dramatic increase in the frequency of genomic insertions by Tf1 was detected in CENP-B mutant cells using an integrase-defective Tf1 element. These results show that in ad- dition to silencing LTR retrotransposons through recuitment of histone deacetylases, CENP-B proteins also suppress homologous recombination of these elements with endogenous LTRs.

Lastly, the authors tested whether this CENP-B-mediated surveillance mechanism could also silence an extinct Tf1 element integrated into the S. pombe genome. ChIP analysis demonstrated that Abp1 and Cbh1 were indeed targeted to a locus where a Tf1 element was inserted into a euchromatic gene, SPAC7D4.08. The CENP-Bs suppressed transcription and transposition of the Tf1 element. Surprisingly, integration of Tf1 altered the sub-cellular localization of the euchromatic gene. FISH analysis revealed that SPAC7D4.08 co-localized with Tf bodies when Tf1 was inserted at this locus.

How might this surveillance mechanism have evolved? The authors propose that since both DNA transposons and LTR retrotransposons are flanked by repeat sequences and given that transposases are known to bind inverted repeats of DNA transposons, then an ancient CENP-B precursor may have evolved the ability to bind a Tf LTR (Figure 1). This new function may have been co-opted by the host for the purpose of controlling retroelement mobility. It is intriguing that $S$. pombe has acquired multiple pathways for retrotransposon surveillance such as that involving the Hip1 histone chaperone protein and recent discoveries involving Hst4-mediated repression of Tf2 [12].

In summary, the Cam et al. study extends our understanding of the role of CENP-B proteins, descendents of DNA transposons, in retrotransposon surveillance in S. pombe. There are several questions raised by this study open for future investigation. For example, it will be interesting to determine whether CENP-Bs interact with additional proteins that are required for surveillance of transposable elements. This study may also have important implications for events described in other organisms. For 


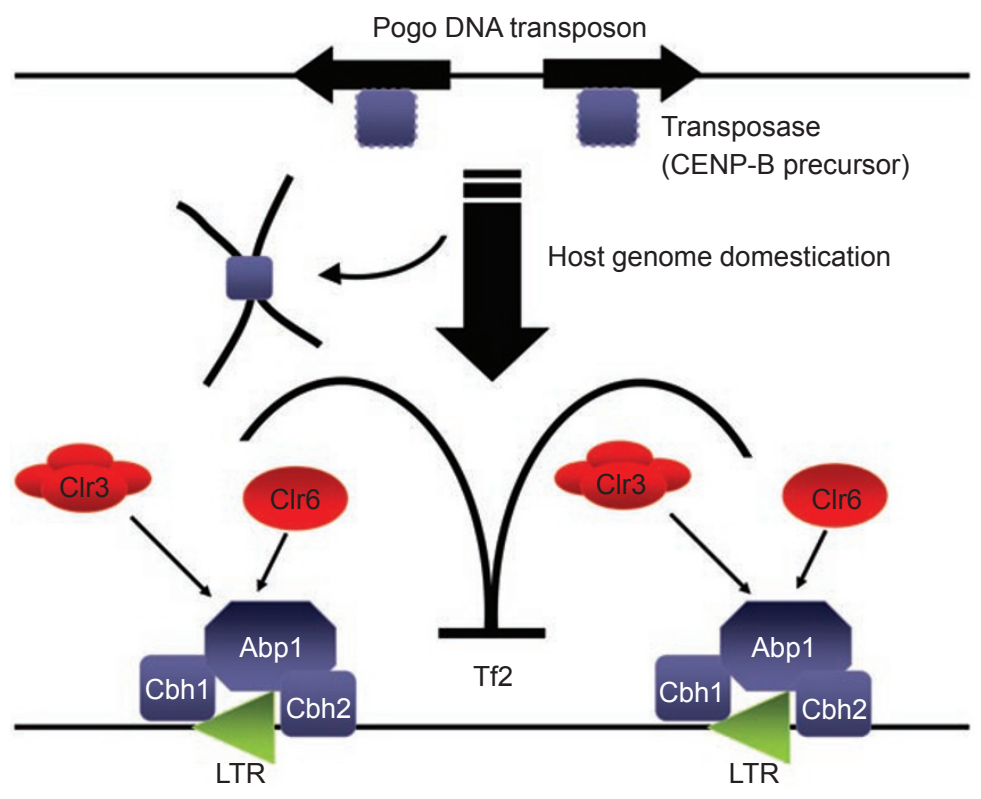

Figure 1 Taming of transposon-derived proteins mediates CENP-B surveillance of Tf2 elements. The CENP-B proteins, Abp1, Cbh1 and Cbh2, are derived from the transposase of pogo DNA transposons. These proteins bind alpha satellite repeats and facilitate centromere formation. During evolution, an ancient CENP-B precursor also acquired the ability to bind Tf long terminal repeats (LTRs). This new function was likely co-opted by $S$. pombe for the purpose of controlling the mobility of Tf retroelements. Cam et al. provide evidence that Abp1 may recruit the other two CENP-B homologues, Cbh1 and Cbh2, to Tf2 LTRs. The Clr3 and CIr6 histone deacetylases, which also have an important role in retrotransposon silencing, may help Abp1 recruit Cbh1 and possibly Cbh2 to Tf2 LTRs.

example in Drosophila, P elements can trigger heterochromatic silencing that is dependent on element copy number. This observation might be analogous to a critical concentration of specific transposon binding proteins such as CENP-Bs, which recruit chromatin modifiers to suppress transposable element expression and recombination. Recently, a novel form of mobile element control has been discovered involving small RNAs in germ cells of higher eukaryotes [13]. However, it is not unlikely that other genome surveillance mechanisms exist in somatic cells, similar to the CENP-based silencing system, which have evolved from an ancestral protein for the benefit of the host organism.

\section{References}

1 Levin HL, Weaver DC, Boeke JD. Two related families of retrotransposons from Schizosaccharomyces pombe. Mol Cell Biol 1990; 10:6791-6798.

2 Cam HP, Sugiyama T, Chen ES, Chen X, FitzGerald PC, Grewal SI. Comprehensive analysis of heterochromatin- and RNAi-mediated epigenetic control of the fission yeast genome. Nat Genet 2005; 37:809-819.

3 Hansen KR, Burns G, Mata J, et al. fission yeast by silencing and RNA interference machineries. Mol Cell Biol 2005; 25:590-601.

4 Cam HP, Noma KI, Ebina H, Levin HL, Global effects on gene expression in
Grewal SI. Host genome surveillance for retrotransposons by transposon-derived proteins. Nature 2008; 451:431-436.

5 Volff JN. Turning junk into gold: domestication of transposable elements and the creation of new genes in eukaryotes. Bioessays 2006; 28:913-922.

6 Irelan JT, Gutkin GI, Clarke L. Functional redundancies, distinct localizations and interactions among three fission yeast homologs of centromere proteinB. Genetics 2001; 157:1191-1203.

7 Nakagawa H, Lee JK, Hurwitz J, et al. Fission yeast CENP-B homologs nucleate centromeric heterochromatin by promoting heterochromatin-specific histone tail modifications. Genes Dev 2002; 16:1766-1778.

8 Murakami Y, Huberman JA, Hurwitz J. Identification, purification, and molecular cloning of autonomously replicating sequence-binding protein 1 from fission yeast Schizosaccharomyces pombe. Proc Natl Acad Sci USA 1996; 93:502507.

9 Greenall A, Williams ES, Martin KA, et al. Hip3 interacts with the HIRA proteins Hip1 and SIm9 and is required for transcriptional silencing and accurate chromosome segregation. $\mathrm{J}$ Biol Chem 2006; 281:8732-8739.

10 Gerasimova TI, Byrd K, Corces VG. A chromatin insulator determines the nuclear localization of DNA. Mol Cell 2000; 6:1025-1035.

11 Atwood A, Choi J, Levin HL. The application of a homologous recombination assay revealed amino acid residues in an LTR-retrotransposon that were critical for integration. J Virol 1998; 72:13241333.

12 Durand-Dubief M, Sinha I, FagerströmBillai $\mathrm{F}$, et al. Specific functions for the fission yeast Sirtuins Hst2 and Hst4 in gene regulation and retrotransposon silencing. EMBO J 2007; 26:2477-2488.

13 Brennecke J, Aravin AA, Stark A, et al. Discrete small RNA-generating loci as master regulators of transposon activity in Drosophila. Cell 2007; 128:10891103. 